\title{
New Measurements of the Surface Ionization at Low Energies
}

\author{
C. Merlet* and X. Llovet** \\ * ISTEEM, CNRS, Université de Montpellier II, Place E Bataillon, 34095 Montpellier, France \\ ** Serveis Cientifico-Tècnics, Universitat de Barcelona, Lluís Solé i Sabarís 1-3, 08028 Barcelona, \\ Spain
}

In electron probe microanalysis (EPMA), the surface ionization $\phi(0)$ describes the increase of ionizations in a thin layer of a given element located at the sample surface due to electrons backscattered from the substrate. Accurate knowledge of this parameter, for incident electron energies from ionization threshold up to $40 \mathrm{keV}$, is of interest for both theoretical and practical uses. On the one hand, $\phi(0)$ appears in most of the analytical parameterizations of the depth distribution of ionizations or $\phi(\rho z)$ function used in quantitative EPMA procedures. While for bulk specimens the evaluated concentrations depend on this parameter especially when $\mathrm{x}$-ray absorption is important, for thin films and multilayers, EPMA results are largely sensitive to the accuracy of the adopted $\phi(0)$ values. On the other hand, the surface ionization is also used in quantitative Auger electron spectroscopy (AES), where it is commonly referred to as the Auger backscattering factor $(\mathrm{r})$, and it describes the increase of the Auger current due to backscattered electrons. Last but not least, the surface ionization is an excellent test to check the reliability of electron transport calculations. Indeed, $\phi(0)$ is not only sensitive to the cross sections adopted to describe elastic scattering and slowing down of electrons but also to the model adopted for inner-shell ionization.

Measurements of the surface ionization have been performed by several groups over the past years. In our previous works $[1,2,3,4]$, we have measured the surface ionization for bulk samples and thinfilm and multilayer samples deposited on a wide variety of substrates. In spite of the experimental uncertainties (of about 5\%) and the limited set of incident electron energies for which $\phi(0)$ was measured, the data obtained made it possible to asses the reliability of $\phi(0)$ calculations at moderately high energies and to guide the development of new analytical formulas. However, it is still difficult to asses the reliability of $\phi(0)$ calculations at very low energies, especially close to the ionization threshold and the need for improved experimental measurements at low energies remains open.

In this communication, new measurements of the surface ionization are presented. We have determined $\phi(0)$ by using the tracer method, i.e. by measuring the characteristic $\mathrm{x}$-ray intensity emitted from an ultra-thin tracer layer deposited on a bulk substrate and, according to Castaing's definition, normalizing it to the intensity emitted from an equivalent, self-supporting tracer layer. Although conceptually simple, the determination of $\phi(0)$ faces numerous difficulties, from sample preparation to the measurement itself. Indeed, the tracer layer should be as thin as possible to minimize multiple-scattering effects (finite-thickness effect), especially at low accelerating voltages, but should be thick enough to yield acceptable signal-to-noise ratios so as to minimize statistical uncertainties and systematic errors in spectral background subtraction. In this work, we have used tracer layers with thicknesses of about $2 \mathrm{~nm}$, deposited on TEM grids. For backing purposes, carbon layers of about $20 \mathrm{~nm}$ thick were previously set on the TEM grids. However, to minimize backscattering effects at very low energies (which would result in a decrease of the $\phi(0)$ value), we have reduced the thickness of the backing carbon films from $20 \mathrm{~nm}$ down to about $5 \mathrm{~nm}$ by ion 
milling. Tracer and substrate elements have also been selected so as to minimize possible x-ray overlapping and characteristic fluorescence effects induced from $\mathrm{x}$ rays coming from the substrate.

Measurements have been performed on a CAMECA SX-100 electron microprobe, equipped with 5 wavelength-dispersive spectrometers. For the purpose of accurately determining the shape of the $\phi(0)$ curve as a function of the incident electron energy, measurements have been performed at electron energies varying from $1.5 \mathrm{keV}$ up to $39 \mathrm{keV}$ in at least $1 \mathrm{keV}$ steps $(0.5 \mathrm{keV}$ for energies close to the ionization threshold). To minimize the effect of stray radiation (coming from the interaction of electrons and $\mathrm{x}$ rays with the supporting grid and the specimen chamber) a specific sampler holder containing a Faraday cup has been used. Moreover, to minimize uncertainties arising from possible layer thickness inhomogeneities and to a lesser extend stray radiation, $\mathrm{x}$ rays have been measured on 5 different self-supporting films located at different positions in the chamber.

Traditionally in EPMA, the spectral background is estimated by linear interpolation of x-ray intensities measured at both sides of the characteristic peak. However, linear interpolation does not take into account effects such as crystal reflectivity or absorption edges resulting from x-ray absorption in the sample or in the detector. Here, accurate background subtraction is crucial to determine the surface ionization. The peak-to-background ratio is typically high for self-supporting tracer layers but extremely low for tracer layers on top of substrates, mainly because of the effect of the substrate bremsstrahlung. To improve background subtraction, we have estimated background intensities by means of a more accurate procedure which combines x-ray measurements on the substrate sample with and without the tracer film [4]. Improvements in both sample preparation and measurement techniques have allowed us to obtain $\phi(0)$ curves, for $L \alpha$ and $K \alpha$ lines of several tracer elements on different substrates, with uncertainties less than $2 \%$.

The experimental results are compared with the results of Monte Carlo (MC) simulation obtained by using the general-purpose MC code PENELOPE [5], together with ionization cross sections computed from the distorted-wave Born approximation [6]. Results are also compared with various analytical $\phi(0)$ expressions commonly used in EPMA and AES.

\section{References}

[1] C. Merlet, X-Ray Spectrom. 21 (1992) 229

[2] C. Merlet et al., Surf. Interface Anal. 19 (1992) 192

[3] X. Llovet et al., Mikrochimica Acta, [suppl.] 15 (1998) 155

[4] C. Merlet et al., X-Ray Spectrom. 33 (2004) 376

[5] F. Salvat et al., OECD/NEA Data Bank, Issy-les-Moulineaux, France (2003)

[6] S. Segui et al., Phys. Rev. A 67 (2003) 062710. 